\title{
FINITE ELEMENT ANALYSIS FOR THE LECTOTYPE OF THE LARGE ELECTRONIC TRUCK SCALE STRUCTURE
}

\author{
Wei Tieping ${ }^{1}$, Zhou Xingyang ${ }^{2}$, Yang Xiaoxiang ${ }^{3}$, Yao Jinhui ${ }^{4}$ \\ ${ }^{1}$ School of Mechanical and Automotive Engineering, Fujian University of Technology, Fuzhou, China, \\ tpwei@fjut.edu.cn \\ ${ }^{2}$ School of Mechanical and Automotive Engineering, Fujian University of Technology, Fuzhou, China, \\ 1224122843@qq.com \\ ${ }^{3}$ School of Mechanical Engineering and Automation, Fuzhou University, Fuzhou, China, yangxx@fzu.edu.cn \\ ${ }^{4}$ Fujian Province Institute of Metrology, Fuzhou, China, yaojinhui2000@126.com
}

\begin{abstract}
The stiffness of truck scales is the main influence factor on its measurement accuracy. Three kinds of SCS-100T large electronic truck scales are different in size arrangement of U-beam. Based on the finite element method, the models of the truck scales were established. The stiffness and strength of the scales were compared on account of the analysis results. The results show that U-beam arrangement of the low at both ends but high in the middle structure for the truck scale not only can save material but also meet the requirement of stiffness. Simulation analysis provides the theoretical basis for the structural design of truck scales.
\end{abstract}

Keywords: electronic truck scale; U-beam; stiffness; finite element analysis

\section{INTRODUCTION}

At present, the structures of the electronic truck scale mainly include three kinds, including i-section steel, channel-section steel and U-section steel. The study found that U-section steel layout structure of the electronic truck scale was considered to be the most compressive capacity and the highest material utilization rate $[1,2]$. Traditionally, the truck scale structure is usually simplified into a simply supporting beam in order to analyse the stiffness of the truck scale structure [3-5]. However, there is a large error in this simplification method. Therefore, to make the calculation results more consistent with the actual model, many studies have been carried out by establishing a complete three-dimensional finite element model of the scale.

The truck scale structure of U-beam structure was optimised, and the optimised mass ratio was reduced by $6.7 \%$ [6]. The truck scale body structure of section steel was replaced with cold-bending groove made of steel plate, which finally made lighter weight, less consumables and its strength and stiffness requirements in mechanical properties can be achieved [7]. In addition, the strength index should be used as an important checking index when the SCS series truck scales was overloaded by simulation analysis [8]. Also, the deformation of single scales in SCS series $120 \mathrm{t}$ truck scales was studied under different loading modes with the finite element method [9]. However, the size of $u$ shaped beam arrangement has a great influence on the stiffness of the scale body, which has not been seen in the literature.

In this paper, the scale body of SCS-100T Ubeam structure is taken as the research object, and the three U-beam size arrangement modes are simulated and calculated to explore the influence of size arrangement mode on the stiffness. Furthermore, the optimal arrangement mode is determined to provide theoretical basis for the transformation of truck scale without weight verification $[10,11]$.

\section{FINITE ELEMENT MODEL OF SCALE STRUCTURE}

\subsection{Model Simplification and Mesh Generation}

The SCS-100T U-beam truck scale body structure produced by a company was taken as the research object, as shown in Figure 1. Generally, a truck scale mainly consists of the load bearing device (scale platform), the weighing display instrument, the weighing sensor, connecting parts, the limit device and junction boxes. First, when the weighing object or the truck is placed on the scale, its gravity is transferred to the weighing sensors through the scale under the action of gravity, which leads to the deformation of the elastomer of the weighing sensor. Then, the strain gauge bridge attached to the elastomer is out of balance and it will output an electrical signal that is proportional to the 
weight value. After that, the electrical signal is amplified by a linear amplifier and conversed to digital signal with $A / D$ conversion. Finally, the weight signal is processed by the microprocessor of the meter and the weight data is displayed directly.

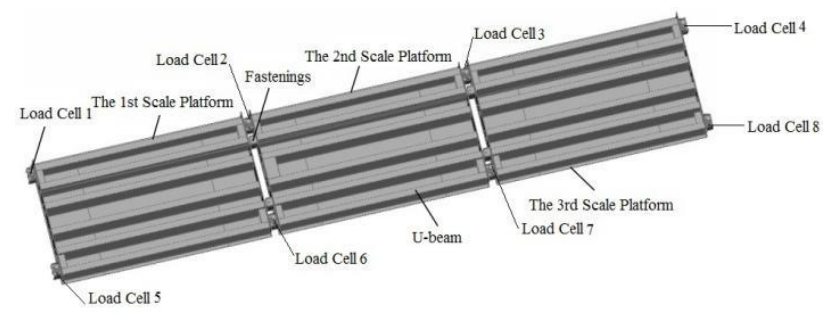

Figure 1: Schematic diagram of the truck scale structure

The layout of three different U-beam size is shown in Figure 2. Figure 2(a) shows that the scale body is designed with equal height layout and the height of the beam is $h$. Figure 2(b) shows that the scale body is designed with the layout of high intermediate and low at two ends. Figure 2(c) shows that the scale body is designed with the layout of low intermediate and high at two ends.

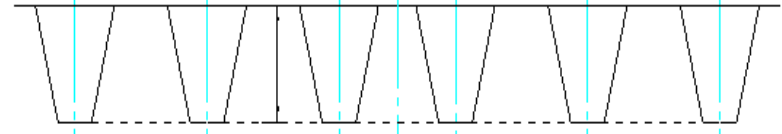

(a) Equal height layout

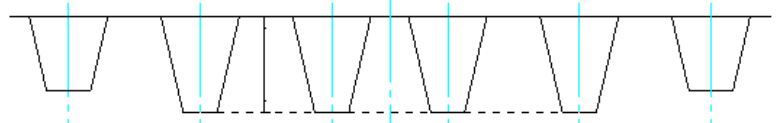

(b) High intermediate and low at two ends layout

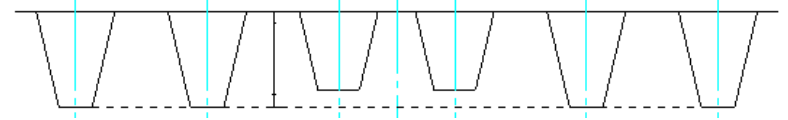

(c) Low intermediate and high at two ends layout

Figure 2: Cross section diagrams of different U-beam size arrangement of the scale body

This study mainly analyses the platform stiffness of different U-beam sizes. Because there is little significant influence of weighing sensors and junction box on the stiffness, it can be ignored. The linear elastic isotropic material Q345 is selected for all components in the structure. Its elastic modulus is $206 \mathrm{GPa}$ and Poisson's ratio is 0.28. In addition, ANSA software is a powerful CAE pre-processing software, which has good efficiency and quality in geometric model and finite element mesh processing. Compared with other similar software, ANSA software has no limit on the size of the model and can read faster. Therefore, the finite element mesh model of the truck scale structure was established with ANSA. The finite element model of the scale structure with U-beam of equal height is shown in Figure 3. Solid 185 element is used as the element type of model. The Solid 185 element is used to construct a three-dimensional solid structure.
The element is defined by eight nodes, each of which has three degrees of freedom translated along the xyz direction. The element has super elasticity, stress toughening, creep, large deformation and large deformation capacity. The element can also simulate almost incompressible elastoplastic materials and completely incompressible super elastic materials using a mixed mode. In the whole model, the longest side length of the cell grid shall not exceed $15 \mathrm{~mm}$, and the shortest is $5 \mathrm{~mm}$ and the numbers of elements and nodes are 377369 and 701895 respectively.

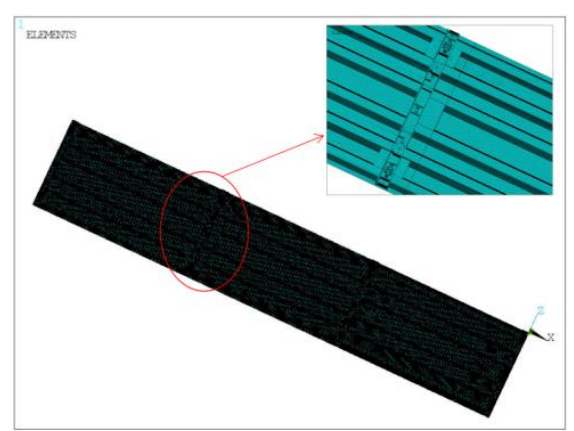

Figure 3: The finite element model of scale structure

\subsection{Boundary Conditions and Loading Conditions}

\section{Boundary Conditions}

In actual working conditions, the structure of the scale body is contacted with the ground through the weighing sensor, and there is no other attachment between the weighing sensor and the ground. Therefore, in the finite element model, the connection between seven load cells and the ground can be simplified as constraining y-direction displacement of load cells' base, while the other one as constraining all degrees of freedom.

\section{Loading Conditions}

According to the standard $G B / T$ 7723-2008 (this standard is a revision of $G B / T$ 7723-2002 Fixed Electronic scale, based on OIML R76 NonAutomatic Weighing Apparatus (2006E) recommended by International Legal Metrological Organization) fixed electronic truck scale on the maximum relative deformation of the large weighing machine, the calibration must be carried out using the load of $40 \mathrm{t}$ acting on the middle of each weighing platform for a scale with a maximum scale of $100 \mathrm{t}$. The loading area is $2.6 \mathrm{~m}$ wide and it can be considered as evenly distributed force.

Boundary constraints and loading conditions are shown in Figure 4. 


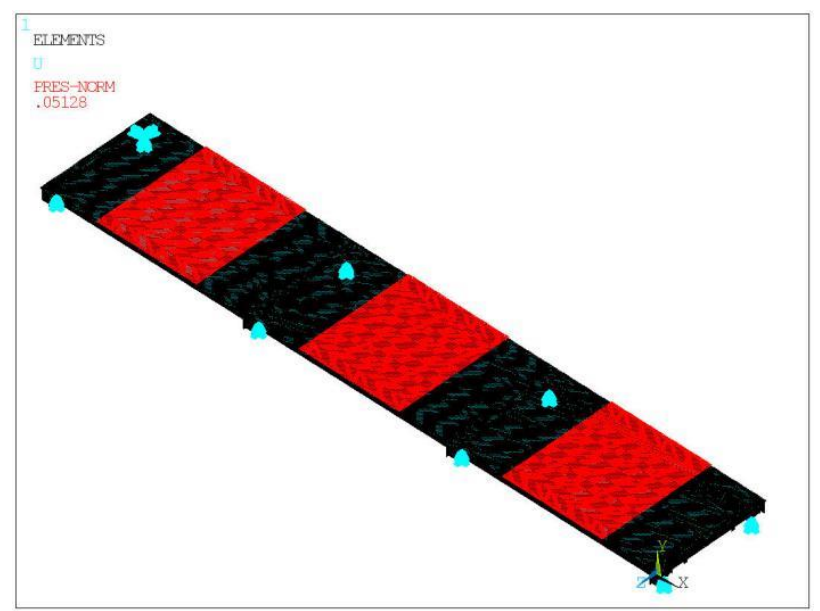

Figure 4: Schematic Diagram of Boundary and Loading Conditions

\section{RESULT OF SIMULATION}

The y-direction displacement distribution cloud diagrams of three different U-beam scales are shown in Figures 5(a), 5(b) and 5(c) respectively. As can be seen from the figures, the main force area of the truck scale is in the middle, and the maximum displacement of the three scales occurs in the centre of the main scale. The maximum displacements values in the y direction of the U-beam scales with equal height layout (as shown in Figure 5 (a)), the U-beam scales with high intermediate and low at two ends layout (as shown in Figure 5 (b)) and the U-beam scales with low intermediate and high at two ends layout (as shown in Figure 5 (c)) are $7.027 \mathrm{~mm}, 7.413 \mathrm{~mm}$ and $8.125 \mathrm{~mm}$, respectively, which all occur in the middle panel of the first scale body.

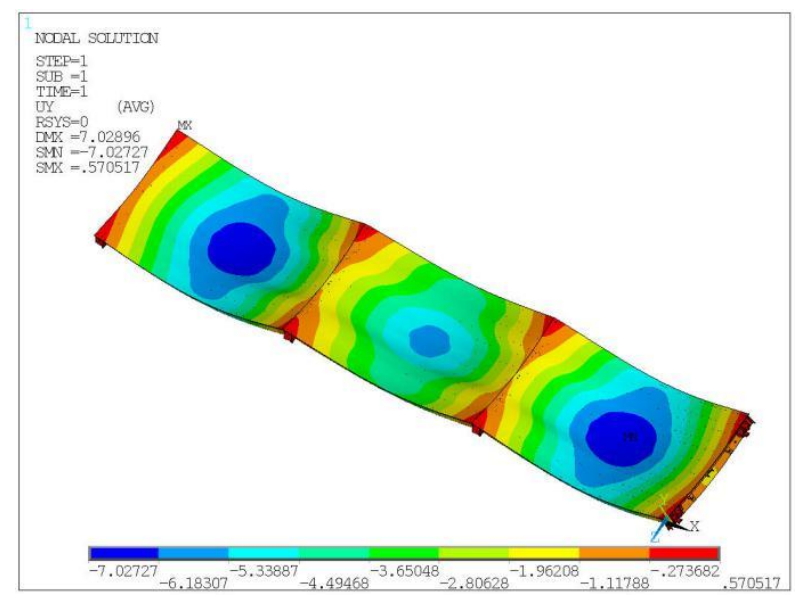

(a) equal height layout

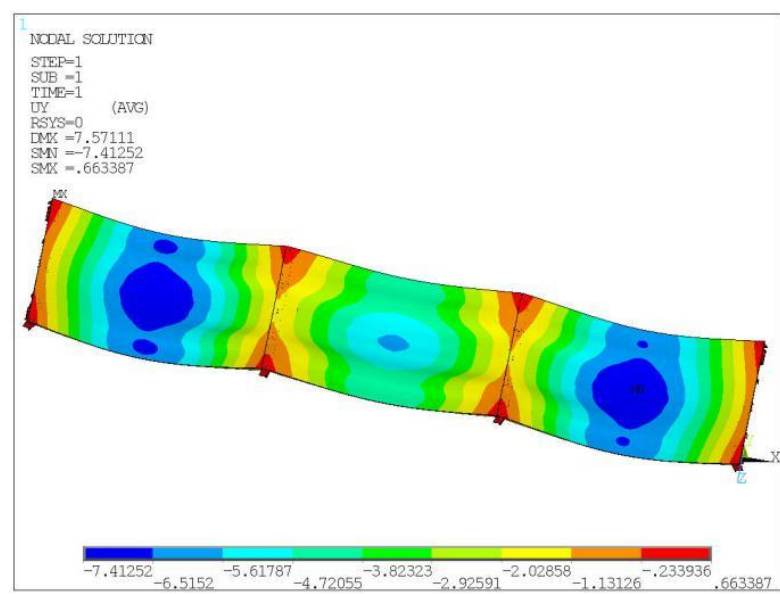

(b) high intermediate and low at two ends layout

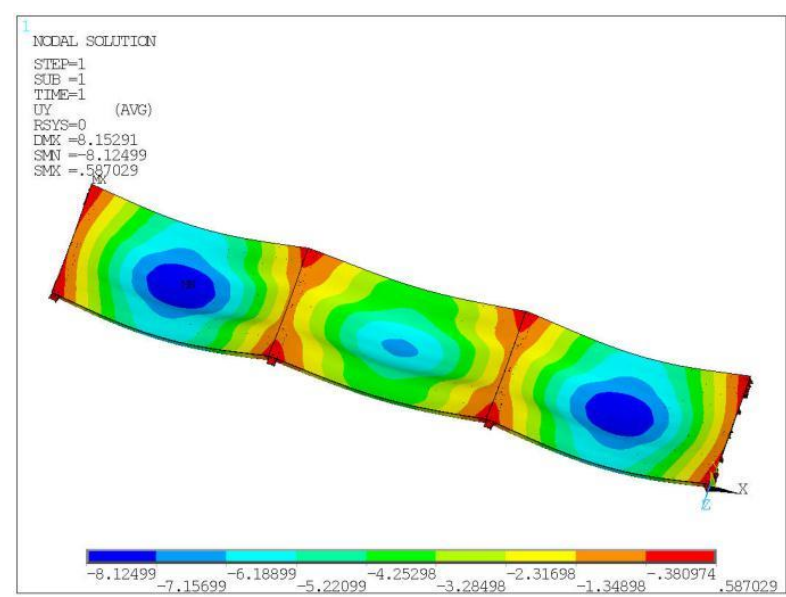

(c) low intermediate and high at two ends layout

Figure 5: The y-direction displacement cloud diagrams of the scale structure with different U-beam

The stress distribution cloud diagrams of three different U-beam scales are shown in Figure 6, Figure 7 and Figure 8, respectively. It can be seen from the figures that the maximum Von Mises stress value of the three scales occurs at the joint of the first scale body and the second scale body. The maximum Von Mises stress values of the three types of scales are in the order from large to small as following: high intermediate and low at two ends layout, low intermediate and high at two ends layout, equal height layout. The maximum stress values are 1221.6 MPa, $1188.6 \mathrm{MPa}$, and $1100.1 \mathrm{MPa}$, respectively. In addition, the maximum stress value positions occur at the joints between the first weighing body and the second weighing body. 


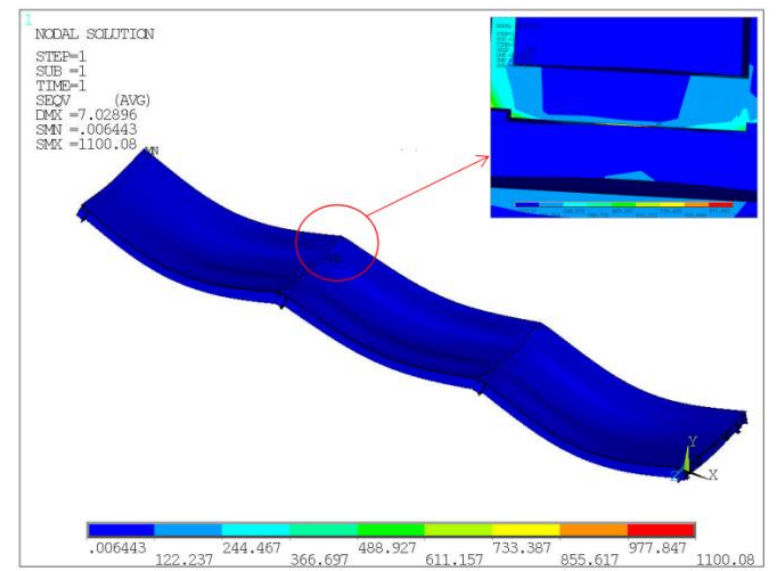

(a) The stress cloud diagram of the whole scale structure

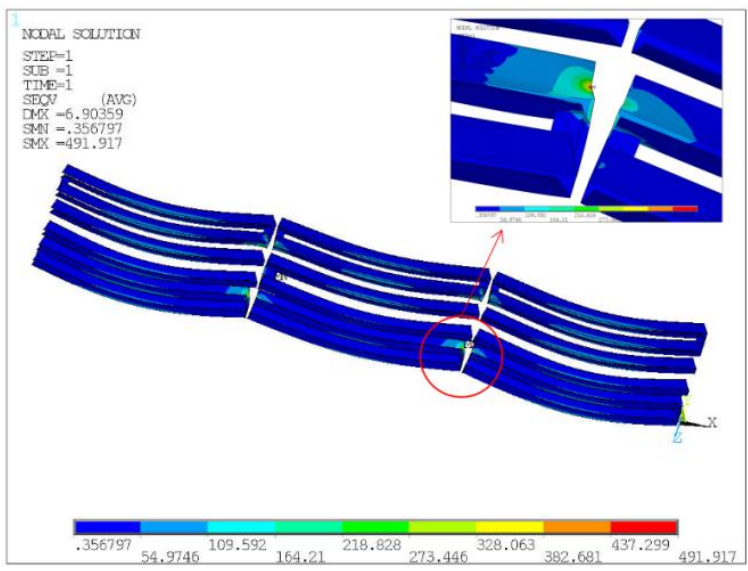

(b) Stress clouds of the U-beam

Figure 6: The Von Mises equivalent stress cloud diagram of the U-beam scale with equal height layout

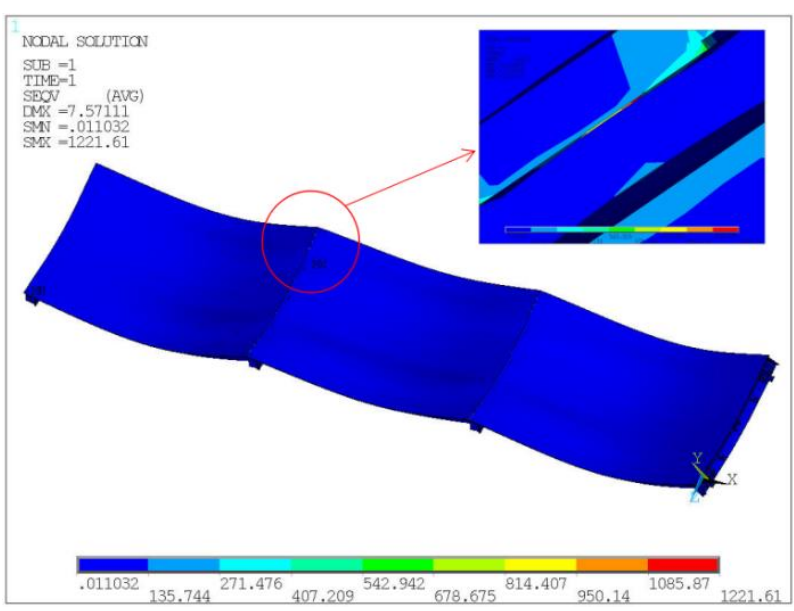

(a) The stress cloud diagram of the whole scale structure

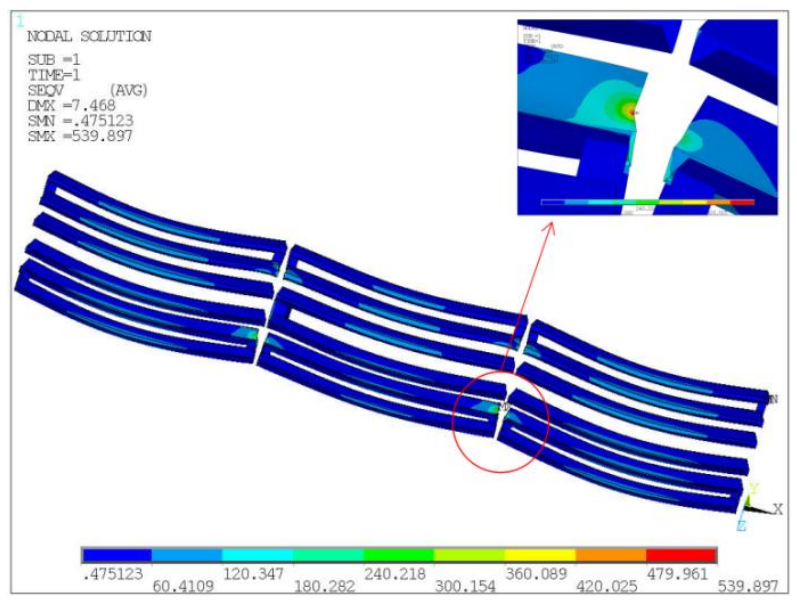

(b) Stress clouds of the U-beam

Figure 7: The Von Mises equivalent stress cloud diagram of the U-beam scale with high intermediate and low at two ends layout

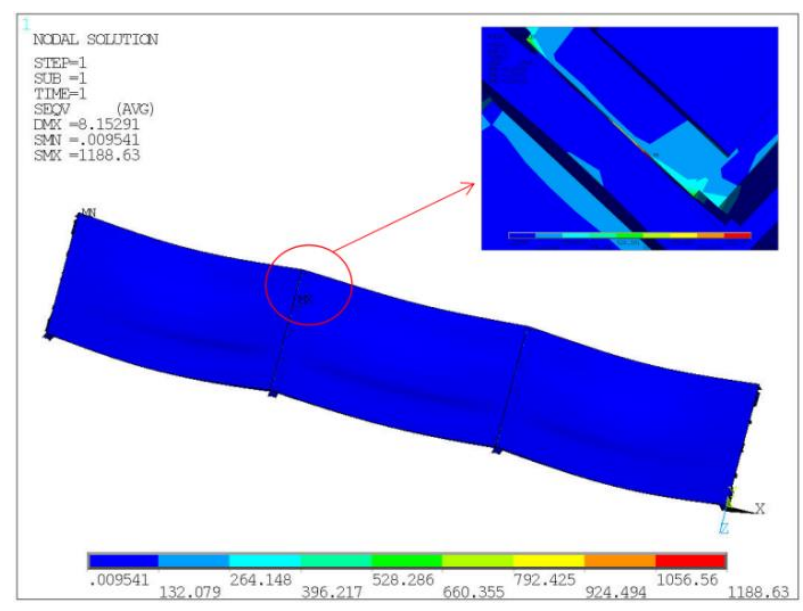

(a) The stress cloud diagram of the whole scale structure

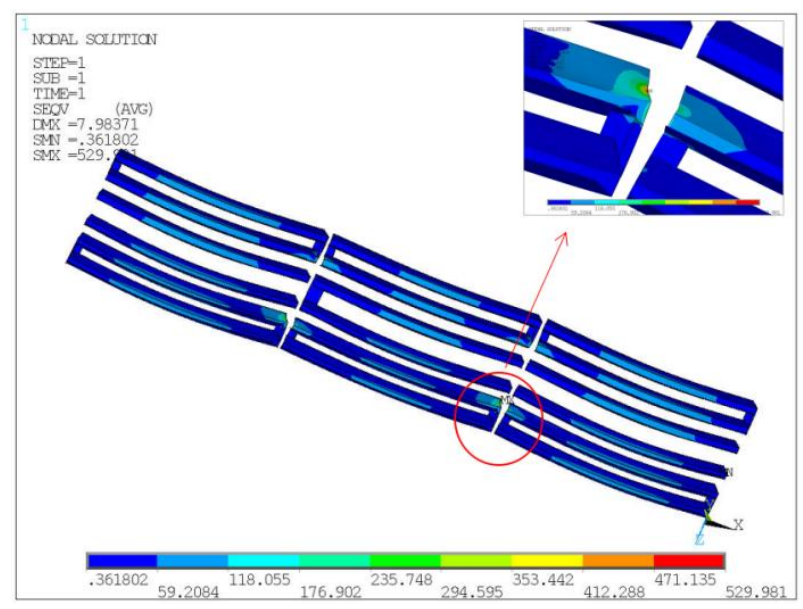

(b) Stress clouds of the U-beam

Figure 8: The Von Mises equivalent stress cloud diagram of the U-beam scale with low intermediate and high at two ends layout

\section{ANALYSIS AND DISCUSSION}

Combining with the analysis from Figure 5 to Figure 8, it can be seen that: (1) the maximum stress value lies at the joint of the scale body's edge, and the stress concentration area at the compression 
point of the U-beam is very small, so it will not affect the strength of the scale body structure; (2) the y-direction displacement of the U-beam with high intermediate and low at two ends layout is larger than that of the U-beam with equal height layout, but the difference is very small. In addition, the scale body is more evenly loaded, and the deflection of the scale body is within the scope of the regulation standard. Therefore, reducing the size of U-beam at both ends can improve the stress state of the structure and achieve the purpose of saving material and cost; and (3) for the U-beam with low intermediate and high at two ends layout, because of reducing in the size of the two U type beam which is mainly loaded, the deflection of the scale increases. According to the regulation GB/T 77232008 fixed electronic truck scale, the U-beam scale with low intermediate and high at two ends layout does not meet the stiffness requirements (the maximum deformation of the weighing body in use shall not exceed 1/600 of the length of the weighing platform, and the corresponding displacement shall not exceed $8 \mathrm{~mm}$ ).

\section{CONCLUSION}

Based on finite element method, the influence of the strength and stiffness of three scales with different U-beam size layout was analysed in this paper. According to the simulation results, the following conclusions and recommendations can be obtained as following:

(1) The structural stiffness of the three scales is in the order from large to small as following: equal height layout, high intermediate and low at two ends layout, low intermediate and high at two ends layout.

(2) The middle part of the scale structure is the main deformation area which is under the maximum load. The scale structure of the U-beam with low intermediate and high at two ends layout is not reasonable and it does not meet the rigidity requirements.

(3) The scale body of the U-beam with high intermediate and low at two ends layout not only needs less material and is more uniform in the stress distribution, but also can meet the requirements of strength and stiffness of the scale structure.

\section{REFERENCES}

[1] Wang Jinqi, "Bearing Structure with Welded Beam of $U$ Type of Section for Electronic Weighing Apparatus of Trucks", Port Operation, no. 3, pp. 37-37, July 2004.

[2] Jiang Binsong, "Analysis of Prestress Force of Yieldable U-steel Support", China Mining Magazine, vol. 7, no. 2, pp. 43-46, April 1998.

[3] Guo Zhiqiagn, Wu Zhanchao, Fang Shaohui, "Structural Design and Analysis for U Type Dynamic Electronic Truck Scale", Machinery Design \& Manufacture, no. 8, pp. 13-15, August 2011.

[4] Wang Zhenrong, "Discussion on the Structure and Stiffness Calculation of the Electronic Truck Scale Platform", Weighing Instrument, no. 1, pp. 24-26, February 1997.

[5] Wang Zhenrong, "Simple Design and Calculation of An Electronic Truck Scale", Weighing Instrument, vol 36, no. 4, pp. 6-10, July 2007.

[6] Lin Yuli, Yang Xiaoxiang, Wei Tieping, "Structural Optimization of U Type SCS-100T Electronic Truck Scale", Journal of Mechanical \& Electrical Engineering, vol 31, no. 3, pp. 346-349, March 2014.

[7] Qi Jjianmin, Gu Jianhua, "One Kind of Improvement Plan About Electronic Truck Scale Body Design", Weighing Instrument, vol 35, no. 6, pp. 26-27, November 2006.

[8] Liu Mei, Zhong Peisi, Sun Zaoyang, Ding Shuhui, "Finite Element Analysis for Modular Platform of SCS Series Truck Scale with Multi-Block", Control \& Automation, vol 20, no. 9, pp. 106-107, September 2004.

[9] W. T. Shi, J. Li, J. He, "Finite element analysis for analog modes of SCS series truck scale with different loads", Advanced Materials Research, vol. 201, pp. 121-125, 2011.

[10] Wei Tieping, Yang Xiaoxiang, Yao Jinhui, Xu Hang, "The Influence of A Balanced Structure on the Rotation Effect of A Build-up System", Measurement, vol. 61, pp. 162-168, February 2015.

[11] Wei Tieping, Yang Xiaoxiang, Yao Jinhui, Xu Hang, "The Additional Side Force on the Force Transducer in the Combinatorial Load Cell", Advanced Materials Research, vol. 541, pp. 13271332, 2014. 